\title{
Evaluation of the Kinetic Property of Single-Molecule Junctions by Tunneling Current Measurements
}

\author{
Takanori Harashima, Yusuke HaSegaWa, Manabu KiguCHI, and Tomoaki Nishino ${ }^{\dagger}$ \\ Department of Chemistry, School of Science, Tokyo Institute of Technology, 2-12-1 Ookayama, Meguroku, \\ Tokyo 152-8551, Japan
}

\begin{abstract}
We investigated the formation and breaking of single-molecule junctions of two kinds of dithiol molecules by timeresolved tunneling current measurements in a metal nanogap. The resulting current trajectory was statistically analyzed to determine the single-molecule conductance and, more importantly, to reveal the kinetic property of the single-molecular junction. These results suggested that combining a measurement of the single-molecule conductance and statistical analysis is a promising method to uncover the kinetic properties of the single-molecule junction.
\end{abstract}

Keywords Single molecule, machine learning, scanning tunneling microscopy, hidden Markov model, molecular junction

(Received May 18, 2018; Accepted May 22, 2018; Published June 10, 2018)

\begin{abstract}
In-situ observations of a chemical reaction on a single-molecule basis can offer a powerful means to directly explore the mechanism and energy dynamics of the reaction. Recently, scanning tunneling microscopy (STM) has enabled such investigations to observe, and even to control, the reactions of a molecular junction, where a molecule bridges a gap between the STM tip and the substrate, at the single-molecule level. ${ }^{1-7}$ For example, observations of the Diels-Alder reaction taking place between single molecules have revealed that this reaction can be catalyzed by an electric field across the nanogap. ${ }^{8}$ Measuring a DNA hybridization reaction between single-stranded DNAs allows for a single-molecule epigenetic analysis. ${ }^{3}$ These investigations of the reaction between single molecules have relied on electrical measurements of the conductance for a single reactant or the product molecule. It can be anticipated that the time course of the conductance, which deviates in accompany with the reaction, provides kinetic information mandatory for examining the reaction mechanism. In the present study, we performed time-resolved measurements of the tunneling current to reveal the kinetic property of singlemolecule junctions. As archetypal examples, two kinds of dithiol molecules, hexanedithiol (HDT) and benzenedithiol (BDT), were investigated. An analysis of the current was performed using the hidden Markov model (HMM). HMM has been utilized to analyze time-series data where signals have a low signal-to-noise ratio. ${ }^{9}$ The single-molecule conductance of an organic molecule is extremely low, typically ranging from $10 \mathrm{pS}$ to $10 \mathrm{nS}$. Furthermore, the difference in the singlemolecule conductance between different, but structurally relevant, molecules, like a reactant and a product of a given reaction, is minute..$^{10,11}$

Au wires (99.999\%, $0.25 \mathrm{~mm}$ diameter) were electrochemically etched to prepare the STM tip. An Au(111) surface, prepared by thermal evaporation, was used as a substrate. The substrates were chemically modified by immersion for $10 \mathrm{~min}$ in a $50 \mu \mathrm{M}$ ethanolic solution of HDT or BDT. STM measurements were
\end{abstract}

$\dagger$ To whom correspondence should be addressed.

E-mail: tnishino@chem.titech.ac.jp performed on an Agilent SPM5100 system. The tunneling current was monitored at a sampling frequency of $20000 \mathrm{~Hz}$ with a bias voltage of $400 \mathrm{mV}$ for HDT or $50 \mathrm{mV}$ for BDT. In a break junction (BJ) measurement, the STM tip was repeatedly brought into and out of contact with the HDT modified surface at $60 \mathrm{~nm} / \mathrm{s}$ while keeping the $x$ and $y$ positions constant. In a current-time (I-t) measurement, the STM tip was first approached to a surface modified with the target molecule at a setpoint current of 50 and $20 \mathrm{nA}$ for HDT and BDT, respectively. After the feedback loop was turned off, the tunneling current was measured for $2 \mathrm{~s}$ with the tip held stationary. The HMM analysis was carried out by the hmmlearn library for Python implementing the Baum-Welch forward-backward algorithm and the Viterbi algorithm. The HMM has been shown to be quite effective for stochastic phenomena at the nanoscale. For example, through an HMM analysis of the fluorescence resonance energy transfer, the probability of a conformational change of a single biomolecule was determined. ${ }^{12,13}$ In nanopore experiments, an HMM analysis revealed the probability of passing of the target molecule through the nanopore.

We first performed an STM-BJ measurement. In this

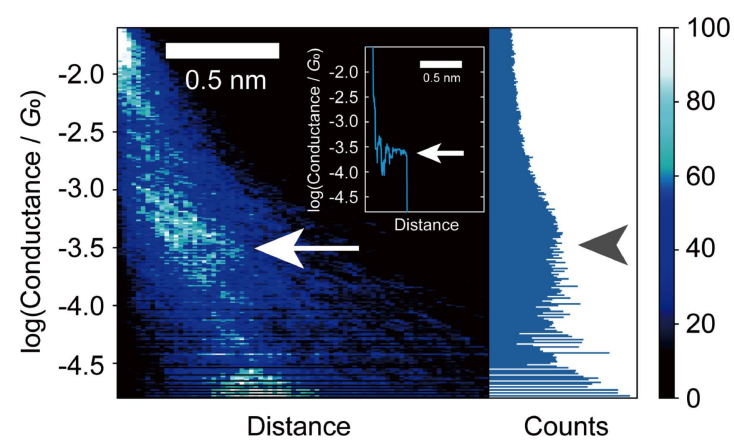

Fig. 1 Two-dimensional histogram of the conductance trace constructed from 1034 traces (left) and one-dimensional conductance histograms of HDT (right). The bin sizes for the distance and $\log \left(\right.$ conductance $\left./ G_{0}\right)$ were $0.023 \mathrm{~nm}$ and 0.021 , respectively. 
a

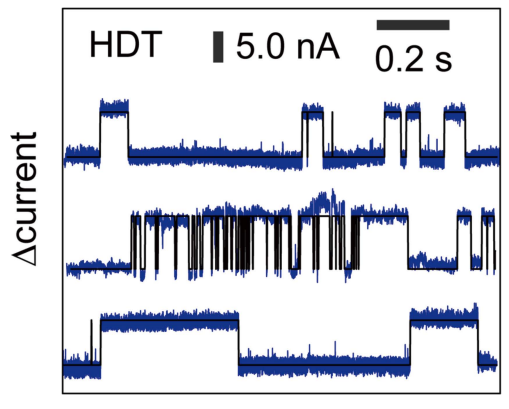

Time b

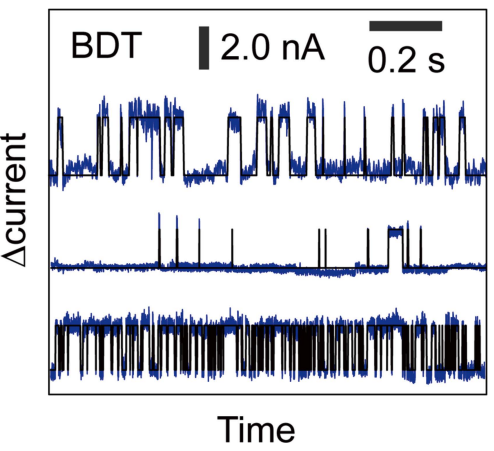

Fig. 2 Representative raw $I-t$ traces (blue) of (a) HDT and (b) BDT. The idealized trajectory obtained by HMM is overlaid (black).

a

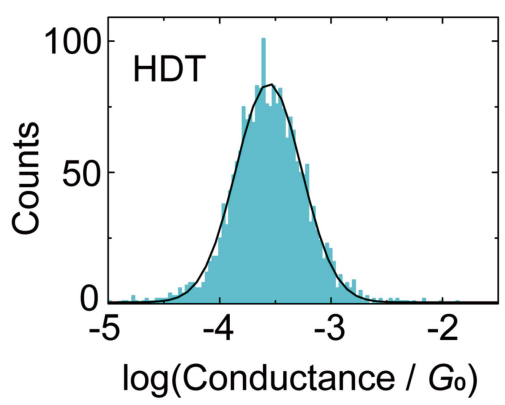

b

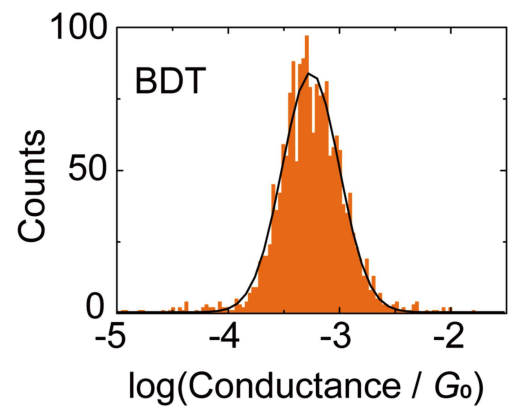

Fig. 3 Conductance histograms for (a) HDT and (b) BDT determined from the HMM analysis of $I-t$ traces. Bin size, 0.03 . The Gaussian fit was overlaid on the histogram with black lines.

technique, the STM tip is brought into contact with a substrate surface modified with a sample molecule. The tip is then pulled up to form a single-molecule junction. The current, from which the conductance is calculated, is recorded during the last pullingup procedure. Figure 1 shows a two-dimensional conductance histogram, where thousands of the conductance traces of HDT were overlaid. The conductance traces, as shown in the inset of Fig. 1, clearly exhibit plateaus, where conductance remains constant, at $10^{-3}-10^{-4} G_{0}\left(1 G_{0}=2 e^{2} / h, e\right.$ and $h$ are elementary charge and Planck constant, respectively). The appearance of the plateau demonstrates the formation of the molecular junctions of HDT. ${ }^{14}$ The conductance traces were transformed into one-dimensional histogram using the conductance value of each data point (Fig. 1, right). This histogram exhibited a pronounced peak corresponding to the statistically most probable conductance at which plateaus appeared. Based on the peak position, the single-molecule conductance of HDT was found to be $(2.6 \pm 0.6) \times 10^{-4} G_{0}$. This value agrees well with previous reports. ${ }^{15}$

Next, I- $t$ measurements were performed. In this technique, the tip is held stationary in close proximity to the sample surface so as to observe any spontaneous formation of a single-molecule junction. The resulting current traces (Fig. 2) show characteristic current jumps and consecutive plateaus with increased current. No such jump was observed on a clean Au substrate. These current jumps were attributed to the formation of a single molecule junction of HDT, in accordance with literature where similar transition events in $I-t$ traces took place by the formation of alkanedithiol molecular junctions. ${ }^{16}$

To estimate the transition current value accurately, $I-t$ traces were analyzed by HMM. In the $I-t$ measurement, there exist two possible states: the molecular junction is either present ("connected") or absent ("disconnected") within the gap between the tip and the substrate. HMM analysis enables inferring that the given current values observed in the $I-t$ traces arise either from the connected or the disconnected state. Furthermore, the analysis gives the distribution of the current values obtained from these states and the transition probabilities between the states.

The results of HMM are illustrated by black lines in Fig. 2, together with the raw I- $t$ trace (blue lines). It can be clearly seen that current transitions caused by the formation and breakdown of the molecular junction were correctly assigned by the HMM analysis. Consequently, the current increase originating from the jump signal in every $I-t$ traces can be determined without any manual data inspection, which could cause a biased analysis. Thousands of traces were analyzed by HMM, and the conductance value, calculated from the increased current, was determined for each jump signal in the traces. The conductance histogram of HDT showed a single peak at $(2.3 \pm$ $0.5) \times 10^{-4} G_{0}$ (Fig. 3a). This conductance value agreed with that determined by the STM-BJ measurement within the experimental error. In order to prove the versatility of this analysis, a similar analysis was performed on the molecular junction of BDT. Figure $3 \mathrm{~b}$ shows conductance histograms of BDT at the peak value of $(6.0 \pm 0.5) \times 10^{-4} G_{0}$, which are in good agreement with a previous report. ${ }^{17}$ Several values for the single-molecule conductance originated from different binding configurations between the molecule and electrodes were also reported. ${ }^{18}$ In contrast, a single conductance value was found in 
a

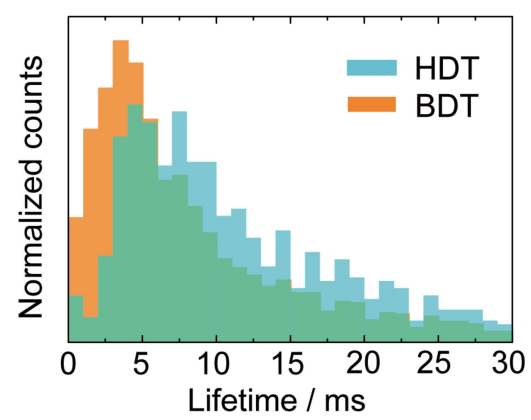

b

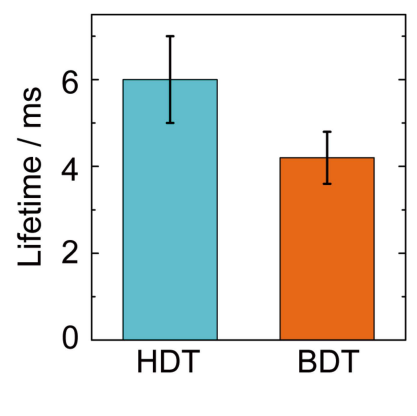

Fig. 4 (a) Lifetime histograms for HDT (cyan) and BDT (orange) determined on the basis of the transition probability of the HMM analysis. Bin size, $1 \mathrm{~ms}$. (b) Bar graph of the lifetime for HDT and BDT.

the present $I-t$ measurements. We attributed this observation to the fixed distance between the tip and the substrate. In such a circumstance, the molecular junction in the most favorable binding configuration would be predominantly formed, thereby hampering the observation of the multiple conductance values.

In addition to the accurate estimation of the conductance, HMM analysis has an advantage in that the probability of transition from one state to another state can be obtained. As a result, we can estimate the lifetime of the molecular junction based on the inverse of the transition probability from the connected to the disconnected states (Fig. 4a). The lifetimes of the HDT and BDT molecular junctions created by STM were determined to be $6 \pm 1$ and $4.2 \pm 0.6 \mathrm{~ms}$, respectively, as shown in Fig. 4. Importantly, the lifetime of the HDT junction is 1.4 times longer than that of the BDT junction (Fig. 4b). In the $I-t$ measurement, the molecular junctions are formed and subsequently broken under the static condition, i.e., without involving the mechanical motion of the STM tip and the substrate. ${ }^{19}$ Since the junction breakdown is a thermally activated process, ${ }^{20}$ the lifetime reflects the thermal stability of the junction. Since both the BDT and the HDT molecules are connected to the electrodes (the substrate and the STM tip) via the common thiolate groups, the difference in the lifetime between BDT and HDT suggests that the flexibility of molecular backbone strongly affects the thermal stability of the molecular junction. This aspect of the $I-t$ technique could be contrasted with the STM-BJ technique, whereas both techniques are useful in terms of determining the single-molecule conductance. Unlike the $I-t$ measurement, a tensile force is exerted on the molecular junction in the STM-BJ measurement, which makes the thermal stability of the junction obscure..$^{21-23}$

In summary, we performed $I-t$ measurements of singlemolecule junctions. The conductances of the molecular junctions were successfully determined. More importantly, it was demonstrated that the stability of the junction can be evaluted by analyzing the current traces by HMM analysis. Unveiling the kinetic properties on a single-molecule basis will lead to in-depth investigations of a chemical reactions and the exploration of novel phenomena thereof.

\section{Acknowledgements}

This work is supported by JSPS KAKENHI Grants 26288070 , $16 \mathrm{~K} 14018$ and $18 \mathrm{H} 02003$.

\section{References}

1. S. W. Hla, L. Bartels, G. Meyer, and K. H. Rieder, Phys.
Rev. Lett., 2000, 85, 2777.

2. Y. Kim, K. Motobayashi, T. Frederiksen, H. Ueba, and M. Kawai, Prog. Surf. Sci., 2015, 90, 85.

3. P. T. Bui, T. Nishino, H. Shiigi, and T. Nagaoka, Chem. Commun., 2015, 51, 1666.

4. T. Nishino, H. Shiigi, M. Kiguchi, and T. Nagaoka, Chem. Commun., 2017, 53, 5212.

5. H. Imada, K. Miwa, M. Imai-Imada, S. Kawahara, K. Kimura, and Y. Kim, Nature, 2016, 538, 364.

6. S. Fujii, M. Ziatdinov, S. Higashibayashi, H. Sakurai, and M. Kiguchi, J. Am. Chem. Soc., 2016, 138, 12142.

7. T. Harashima, C. Kojima, S. Fujii, M. Kiguchi, and T. Nishino, Chem. Commun., 2017, 53, 10378.

8. A. C. Aragones, N. L. Haworth, N. Darwish, S. Ciampi, N. J. Bloomfield, G. G. Wallace, I. Diez-Perez, and M. L. Coote, Nature, 2016, 531, 88.

9. S. R. Eddy, Nat Biotechnol., 2004, 22, 1315.

10. T. Nishino, N. Hayashi, and P. T. Bui, J. Am. Chem. Soc., 2013, 135, 4592.

11. T. Kozakai, T. Harashima, M. Kiguchi, and T. Nishino, Anal. Sci., 2018, 34, 521.

12. S. A. McKinney, C. Joo, and T. Ha, Biophys. J., 2006, 91, 1941.

13. S. Subramanyam, M. Ismail, I. Bhattacharya, and M. Spies, Proc. Natl. Acad. Sci. U. S. A., 2016, 113, E6045.

14. B. Q. Xu and N. J. Tao, Science, 2003, 301, 1221.

15. M. D. Fu, W. P. Chen, H. C. Lu, C. T. Kuo, W. H. Tseng, and C. H. Chen, J. Phys. Chem. C, 2007, 111, 11450.

16. W. Haiss, R. J. Nichols, H. van Zalinge, S. J. Higgins, D. Bethell, and D. J. Schiffrin, Phys. Chem. Chem. Phys., 2004, 6, 4330.

17. Y. Kim, T. Pietsch, A. Erbe, W. Belzig, and E. Scheer, Nano Lett., 2011, 11, 3734.

18. S. Kaneko, D. Murai, S. Marques-Gonzalez, H. Nakamura, Y. Komoto, S. Fujii, T. Nishino, K. Ikeda, K. Tsukagoshi, and M. Kiguchi, J. Am. Chem. Soc., 2016, 138, 1294.

19. Z. F. Huang, F. Chen, P. A. Bennett, and N. J. Tao, J. Am. Chem. Soc., 2007, 129, 13225.

20. Z. F. Huang, B. Q. Xu, Y. C. Chen, M. Di Ventra, and N. J. Tao, Nano Lett., 2006, 6, 1240.

21. B. Q. Xu, X. Y. Xiao, and N. J. Tao, J. Am. Chem. Soc., 2003, 125, 16164.

22. K. Yoshida, I. V. Pobelov, D. Z. Manrique, T. Pope, G. Meszaros, M. Gulcur, M. R. Bryce, C. J. Lambert, and T. Wandlowski, Sci. Rep., 2015, 5, 9002.

23. W. Hong, D. Z. Manrique, P. Moreno-Garcia, M. Gulcur, A. Mishchenko, C. J. Lambert, M. R. Bryce, and T. Wandlowski, J. Am. Chem. Soc., 2012, 134, 2292. 